\title{
Effect of optical aberrations on intraocular pressure measurements using a microscale optical implant in ex vivo rabbit eyes
}

Samuel J. Han

Haeri Park

Jeong Oen Lee

Hyuck Choo 


\title{
Effect of optical aberrations on intraocular pressure measurements using a microscale optical implant in ex vivo rabbit eyes
}

\author{
Samuel J. Han, ${ }^{\mathrm{a}, \dagger}$ Haeri Park, ${ }^{\mathrm{a}, \dagger}$ Jeong Oen Lee, ${ }^{\mathrm{a}, \mathrm{b}}$ and Hyuck Choo ${ }^{\mathrm{a}, \mathrm{b}, *}$ \\ ${ }^{a}$ California Institute of Technology, Department of Medical Engineering, Pasadena, California, United States \\ ${ }^{\mathrm{b} C}$ California Institute of Technology, Department of Electrical Engineering, Pasadena, California, United States
}

\begin{abstract}
Elevated intraocular pressure (IOP) is the only modifiable major risk factor of glaucoma. Recently, accurate and continuous IOP monitoring has been demonstrated in vivo using an implantable sensor based on optical resonance with remote optical readout to improve patient outcomes. Here, we investigate the relationship between optical aberrations of ex vivo rabbit eyes and the performance of the IOP sensor using a custom-built setup integrated with a Shack-Hartmann sensor. The sensor readouts became less accurate as the aberrations increased in magnitude, but they remained within the clinically acceptable range. For root-mean-square wavefront errors of 0.10 to $0.94 \mu \mathrm{m}$, the accuracy and the signal-to-noise ratio were $0.58 \pm 0.32 \mathrm{~mm} \mathrm{Hg}$ and $15.57 \pm 4.85 \mathrm{~dB}$, respectively. () The Authors. Published by SPIE under a Creative Commons Attribution 3.0 Unported License. Distribution or reproduction of this work in whole or in part requires full attribution of the original publication, including its DOI. [DOI: 10.1117/1. JBO.23.4.047002]
\end{abstract}

Keywords: medical devices; optics; aberration; light; glaucoma.

Paper 180037R received Jan. 18, 2018; accepted for publication Mar. 23, 2018; published online Apr. 12, 2018.

\section{Introduction}

Glaucoma is a leading cause of irreparable blindness, ${ }^{1,2}$ but the underlying mechanism of its pathophysiological development and progression remains unclear. Because the major identifiable and manageable risk factor of the disease is elevated intraocular pressure (IOP), all glaucoma treatments focus on monitoring and reducing elevated IOP ${ }^{3-6}$ However, IOPs are monitored only a few times a year in clinics using tonometry despite its critical role in glaucoma management. Recently, researchers have developed radio-frequency (RF)-based contact-lens IOP sensors for 24-h monitoring and implantable RF-based sensors for direct IOP and other physiological pressure measurements. ${ }^{7-33}$ In addition, an increasing number of optics-based IOP and other biological pressure monitoring have been demonstrated based on flexible photonic crystal, ${ }^{34}$ interferometry, ${ }^{35-42}$ aberrometry, ${ }^{43}$ microfluidic or micromechanical implants, ${ }^{44,45}$ and laser-excited fluorescence. $^{46}$

To provide more accurate and frequent IOP monitoring and to improve treatment outcomes, researchers recently demonstrated implantable IOP sensors with remote optical readout in long-term in vivo studies. ${ }^{47-49}$ The sensor is a hermetically sealed micro-optical cavity with a top surface made of a flexible, transparent $\mathrm{Si}_{3} \mathrm{~N}_{4}$ membrane and a bottom surface made of a mirror-like silicon substrate. The top membrane deflects according to the ambient pressure, changing the resonance of the sensor cavity that is determined by the distance between the top membrane and the bottom mirror surface [Figs. 1(a)-1(c)]. When probed using broadband near-infrared (NIR) light, the sensor reflects an optical resonance spectrum made of peaks and valleys, the locations of which correlate with the present

\footnotetext{
*Address all correspondence to: Hyuck Choo, E-mail: hchoo@caltech.edu
}

†These authors contributed equally to this work. cavity gap and the corresponding IOP value [Fig. 1(c)]. Because NIR light is used to excite the cavity and obtain the pressure readout, the performance of the sensing system is inevitably influenced by optical aberrations that originate from the refractive-index profiles of the cornea and the anterior chamber of the eye. Although such optical aberrations and their influence on the performance of intraocular lenses have been characterized extensively using Shack-Hartmann ( $\mathrm{SH}$ ) sensors in previous clinical studies, ${ }^{50-56}$ the relationship between the performance of the implantable optical IOP sensor and ocular optical aberrations has yet to be studied. Clearly understanding the relationship between the ocular aberrations and the quality of IOP monitoring could ensure the effective and proper use of implantable optical IOP sensors across diverse patient groups.

In this study, we characterized the optical aberrations present in the corneas and anterior chambers of ex vivo rabbit eyes and examined their influence on the accuracy and signal-to-noise ratio (SNR) of IOP monitoring using optical-resonance-based sensor implants. The aberrated wavefronts of the optical reflection from an implanted sensor are related to the distortion and dislocation of a focal point, which in turn affects the accuracy and SNR of optical IOP monitoring. A customized setup was developed by coaxially integrating an SH sensor and a commercially available spectrometer to measure consecutively both the optical aberrations of the ex vivo eyes and the reflected optical spectra from the implanted IOP sensors [Fig. 1(d)]. First, we implanted a well-characterized reference chip coated with a thin photoresist film in ex vivo rabbit eyes and measured the optical spectra and the wavefront aberrations consecutively; doing so revealed the influence of the aberrations on the accuracy and SNR of the optical spectra obtained from the implanted reference chip. Next, we implanted the optical IOP sensors in $e x$ vivo rabbit eyes, performed the same measurements, and determined the influence of the aberrations on the accuracy and SNR 
of IOP measurements. Finally, we investigated the effect of the position-dependent aberrations on IOP measurements by implanting the sensors at multiple locations inside an ex vivo rabbit eye and correlating IOP with spectral measurements.

\section{Methods}

\subsection{Experimental Setup and Calibration}

Figure 1(d) shows the experimental setup customized for ex vivo rabbit-eye measurements in this study. The setup enabled consecutive measurements of optical spectra and aberrations by incorporating two different systems: (1) a commercially available NIR spectrometer (Maya 2000; Ocean Optics, Dunedin, Florida) operating at 800 to $1100 \mathrm{~nm}$ with a broadband (400 to $1300 \mathrm{~nm}$ ) halogen fiber-optic light source (OSL1; Thorlabs, Newton, New Jersey) for spectral resonance measurements and (2) a $150-\mu \mathrm{m}$-pitch CMOS-based SH sensor (WFS20-5C; Thorlabs, Newton, New Jersey) with a 940-nm laser diode (LP940-SF30; Thorlabs, Newton, New Jersey) for aberration characterization. Both the broadband light and the 940-nm laser were collimated and aligned coaxially to form focal points identical in size and location on the implant under measurement. The two distinct measurements, namely spectral and aberration, shared the same optical path, including an achromatic (690 to $1200 \mathrm{~nm}$ ) quarter-wave plate (AQWP05M-980; Thorlabs, Newton, New Jersey), a 0.55-NA objective lens (50× M PLAN APO; Mitutoyo, Japan), the cornea of the ex vivo rabbit eye, the anterior chamber, and the active area of the sensor. A $0.55-\mathrm{NA}$ objective provided a focal spot size that matched the active area of the sensor. Therefore, we were able to obtain the aberration data only from the active area of the sensor and the corneal area right above it.

Before use, the integrated SH sensor was precharacterized to remove any aberrations that could originate from the objective lens and other optical components in the experimental setup; this was done using a polished silicon chip immersed in water as a reflective surface for double-pass calibration. After calibration, the experimental setup showed an accuracy of $9.317 \mathrm{~nm}$ or approximately $\lambda / 101$, which is very close to the accuracy of $\lambda / 100$ specified by the manufacturer. (a)

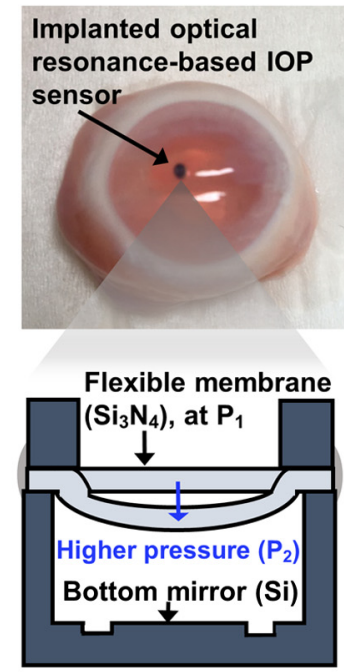

(c)

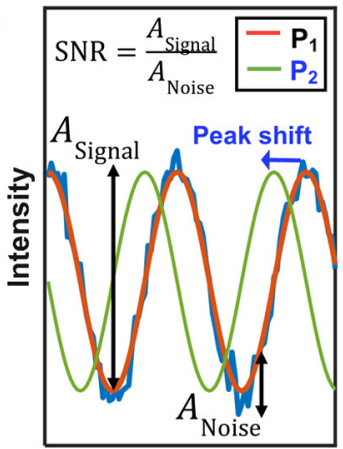

Wavelength (d)

\section{Broadband light path \\ Laser diode light path}

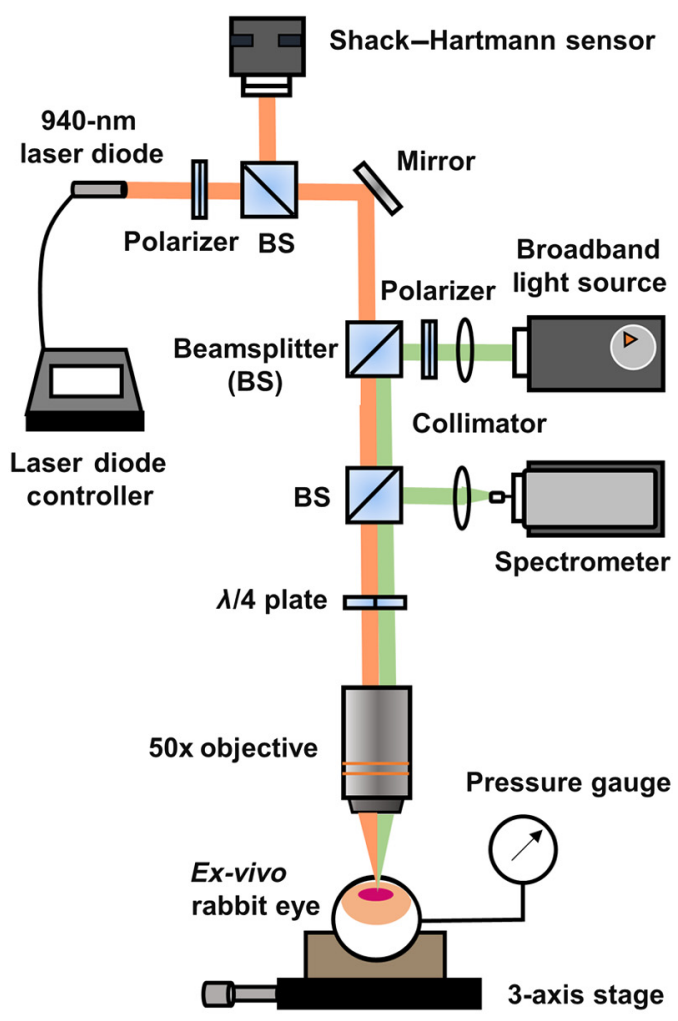

Fig. 1 (a) A photograph of an ex vivo rabbit eye after IOP-sensor implantation. (b) A cross-sectional illustration of the hermetically sealed optical IOP sensor: an increase in IOP from $P_{1}$ to $P_{2}$ deflects the flexible membrane and changes the cavity's optical resonance. (c) The optical resonance spectra reflected from the IOP sensor: the increase in IOP from $P_{1}$ to $P_{2}$ blueshifts the resonance, and there is one-to-one mapping between the resonance spectrum and the corresponding IOP. The accuracy is calculated by extracting the peak position, retrieving an IOP value, and comparing with the pressure-gauge readout. The SNR is calculated by extracting the amplitudes of the signal and noise and calculating the ratio between the two. (d) An illustration of the experimental setup for measuring optical aberrations and resonance spectra. Two light sources, the 940-nm laser diode (orange) and the broadband light source (green), share most of the optical path that includes an objective lens and an ex vivo rabbit eye. 


\subsection{Measurements and Analysis}

To study the influence of the optical aberrations on the sensor performance, we obtained one spectral and five aberration measurements in sequence and grouped them as one set. For the spectral measurement, we used only the broadband light source and collected an optical spectrum ranging from 800 to $1100 \mathrm{~nm}$ from an implant through the integrated spectrometer every $100 \mathrm{~ms}$ at a resolution of $0.22 \mathrm{~nm}$. The sensor used in the experiments showed two peaks within the bandwidth of the collected spectrum. After collecting the optical spectra, we turned off the broadband light source and turned on the laser diode to perform five consecutive aberration measurements every $1 \mathrm{~s}$ at a frame rate of $79 \mathrm{fps}$. Between each set of measurements, we irrigated the ex vivo rabbit eyes with saline solution to maintain a uniform tear film.

We first focused on investigating the fundamental relationship between optical aberrations and the quality of the detected optical spectra using a reference chip that generated a well-characterized static optical spectrum similar to that of the implantable sensor. This eliminated the influence of ambient pressure or variation in the sensor geometry on the measurements. This reference chip was prepared by coating a silicon wafer with $15-\mu$ m-thick photoresist (Microchemicals $\mathrm{GmbH}$, Germany) and dicing it into $1-\mathrm{mm} \times 1-\mathrm{mm}$ chips. The static, unchanging resonance came from the photoresist layer. Ten of these chips were inserted into 10 ex vivo rabbit eyes (Pel-Freeze, Rogers, Arkansas) through a 1-mm incision on the side of the corneas and placed at the center of the anterior chamber.

Next, we studied the effects of the aberrations on the quality of IOP readout using the aforementioned implantable IOP sensors with a micro-optical cavity. Before the experiments were performed, each sensor was characterized and calibrated using the 0.55 -NA objective to remove any readout errors that could originate from the experimental setup. A sensor mounted on a flexible strip was implanted through a 3-mm incision on the side of the cornea and positioned at the center of the anterior chamber of 10 ex vivo rabbit eyes [Fig. 1(a)], whereupon we sutured the incision to prevent leakage. A commercially available high-accuracy $( \pm 0.26 \mathrm{~mm} \mathrm{Hg})$ digital pressure gauge $(3584 \mathrm{~K} 11$; McMasterCarr, Elmhurst, Illinois) was connected to a 21-gauge needle that in turn was inserted directly into the anterior chambers of the ex vivo eyes to provide reference IOP values. The first five eyes (\#1 to 5) were measured without injection of saline solution, whereas the last five eyes (\#6 to 10) were injected with saline solution to emulate the IOP levels observed in living rabbits, resulting in IOP values of 16 to $20 \mathrm{~mm} \mathrm{Hg}$.

We also examined how the location of the IOP sensor in the eye would change the optical aberrations, which would in turn influence the accuracy and SNR of the IOP sensor. Three IOP sensors were implanted and positioned radially at three different locations inside the anterior chamber of an ex vivo rabbit eye. After the measurements of each eye, a reference chip or sensor was retrieved and rinsed in isopropyl alcohol and deionized water for reuse and the used ex vivo rabbit eye was disposed.

The optical spectra obtained from the sensor measurements were analyzed using a custom-built MATLAB ${ }^{\mathrm{TM}}$ signal processing algorithm that detects the location of the peaks and valleys and calculates the corresponding IOP value. ${ }^{47,49}$ The aberration-measurement data were processed using the software provided with the SH sensor (Thorlabs, Newton, New Jersey). We included tip and tilt in our analysis because they play an important role in the resonance of optical cavities. The root-mean-square (rms) wavefront error was calculated by averaging the values of the lowest 21 Zernike coefficients (representing up to the fifth Zernike order) obtained in the five consecutive measurements. The Zernike orders and terms used in our work are summarized in Table $1 .{ }^{57}$

Table 1 Zernike coefficients of the first five orders. ${ }^{a}$

\begin{tabular}{|c|c|c|c|}
\hline $\begin{array}{l}\text { Zernike } \\
\text { order }\end{array}$ & $\begin{array}{c}\text { Radial/ } \\
\text { azimuthal } \\
\text { degree }(n, m)\end{array}$ & Expression & Aberration \\
\hline \multirow[t]{2}{*}{ First } & $(1,-1)$ & $2 \rho \sin (\theta)$ & Tilt \\
\hline & $(1,1)$ & $2 \rho \cos (\theta)$ & Tip \\
\hline \multirow[t]{3}{*}{ Second } & $(2,0)$ & $\sqrt{3}\left(2 \rho^{2}-1\right)$ & Defocus \\
\hline & $(2,-2)$ & $\sqrt{6} \rho^{2} \sin (2 \theta)$ & $\begin{array}{l}\text { Oblique } \\
\text { astigmatism }\end{array}$ \\
\hline & $(2,2)$ & $\sqrt{6} \rho^{2} \cos (2 \theta)$ & $\begin{array}{c}\text { Vertical } \\
\text { astigmatism }\end{array}$ \\
\hline \multirow[t]{4}{*}{ Third } & $(3,-1)$ & $\sqrt{8}\left(3 \rho^{3}-2 \rho\right) \sin (\theta)$ & Vertical coma \\
\hline & $(3,1)$ & $\sqrt{8}\left(3 \rho^{3}-2 \rho\right) \cos (\theta)$ & $\begin{array}{l}\text { Horizontal } \\
\text { coma }\end{array}$ \\
\hline & $(3,-3)$ & $\sqrt{8} \rho^{3} \sin (3 \theta)$ & Vertical trefoil \\
\hline & $(3,3)$ & $\sqrt{8} \rho^{3} \cos (3 \theta)$ & Oblique trefoil \\
\hline \multirow[t]{5}{*}{ Fourth } & $(4,0)$ & $\sqrt{5}\left(6 \rho^{4}-6 \rho^{2}+1\right)$ & $\begin{array}{l}\text { Primary } \\
\text { spherical }\end{array}$ \\
\hline & $(4,-2)$ & $\sqrt{10}\left(4 \rho^{4}-3 \rho^{2}\right) \sin (2 \theta)$ & $\begin{array}{c}\text { Oblique } \\
\text { secondary } \\
\text { astigmatism }\end{array}$ \\
\hline & $(4,2)$ & $\sqrt{10}\left(4 \rho^{4}-3 \rho^{2}\right) \cos (2 \theta)$ & $\begin{array}{c}\text { Vertical } \\
\text { secondary } \\
\text { astigmatism }\end{array}$ \\
\hline & $(4,-4)$ & $\sqrt{10} \rho^{4} \sin (4 \theta)$ & $\begin{array}{l}\text { Oblique } \\
\text { quadrafoil }\end{array}$ \\
\hline & $(4,4)$ & $\sqrt{10} \rho^{4} \cos (4 \theta)$ & $\begin{array}{l}\text { Vertical } \\
\text { quadrafoil }\end{array}$ \\
\hline \multirow[t]{6}{*}{ Fifth } & $(5,-1)$ & $\sqrt{12}\left(10 \rho^{5}-12 \rho^{3}+3 \rho\right) \sin (\theta)$ & $\begin{array}{c}\text { Vertical } \\
\text { secondary } \\
\text { coma }\end{array}$ \\
\hline & $(5,1)$ & $\sqrt{12}\left(10 \rho^{5}-12 \rho^{3}+3 \rho\right) \cos (\theta)$ & $\begin{array}{l}\text { Horizontal } \\
\text { secondary } \\
\text { coma }\end{array}$ \\
\hline & $(5,-3)$ & $\sqrt{12}\left(5 \rho^{5}-4 \rho^{3}\right) \sin (3 \theta)$ & $\begin{array}{l}\text { Vertical } \\
\text { secondary } \\
\text { trefoil }\end{array}$ \\
\hline & $(5,3)$ & $\sqrt{12}\left(5 \rho^{5}-4 \rho^{3}\right) \cos (3 \theta)$ & $\begin{array}{l}\text { Oblique } \\
\text { secondary } \\
\text { trefoil }\end{array}$ \\
\hline & $(5,-5)$ & $\sqrt{12} \rho^{5} \sin (5 \theta)$ & $\begin{array}{l}\text { Vertical } \\
\text { pentafoil }\end{array}$ \\
\hline & $(5,5)$ & $\sqrt{12} \rho^{5} \cos (5 \theta)$ & $\begin{array}{l}\text { Oblique } \\
\text { pentafoil }\end{array}$ \\
\hline
\end{tabular}

${ }^{\mathrm{a}}$ From Ref. 57. 


\section{Results}

\subsection{Accuracy and SNR of Optical Spectra From the Reference Chip Implanted in Ex Vivo Eyes}

We evaluated the accuracy and SNR of the optical spectra reflected from the reference chips implanted in ex vivo rabbit eyes. Figure 2(a) shows the rms-wavefront error obtained in double-pass aberration measurements reflecting off the reference chips. The rms-wavefront errors were 0.10 to $0.26 \mu \mathrm{m}$. When spectral measurements were made on the reference chips implanted in ex vivo eyes, the locations of the resonance peaks deviated by $2.33 \pm 1.41 \mathrm{~nm}$ from the reference-chip measurements made in water [Fig. 2(b)]. We observed no significant correlation between the total rms-wavefront error and the deviation in resonance-peak positions. When we analyzed the deviation in terms of the rms-wavefront error of each Zernike order, the first-order Zernike coefficients (tip and tilt) had the strongest correlation to the peak-location error [Figs. 2(c) and 2(d)]. The SNR of the obtained spectra from the implanted reference chips showed a negative correlation with the rms-wavefront error, as shown in Fig. 2(e). We observed no single dominant Zernike coefficient that was strongly correlated with the SNR. Even the optical spectrum obtained in the presence of the highest rms-wavefront error $(0.26 \mu \mathrm{m})$ still showed a robust SNR of $22.8 \mathrm{~dB}$, well above the required $15-\mathrm{dB}$ minimum SNR for IOP measurements.

\subsection{Accuracy and SNR of Optical Spectra From an Optical Cavity Implant}

We studied the accuracy and SNR of the optical resonant spectra from the optical-resonance-based IOP-sensing implants in $10 \mathrm{ex}$ vivo rabbit eyes. While the pressure of five ex vivo rabbit eyes (\#1 to 5) was maintained at their original values of $<6 \mathrm{~mm} \mathrm{Hg}$, the other five ex vivo rabbit eyes (\#6 to 10) were injected with saline, resulting in pressures of 10 to $17 \mathrm{~mm} \mathrm{Hg}$ [Fig. 3(a)]. The rms-wavefront errors of the ex vivo eyes with saline injection were 0.38 to $0.94 \mu \mathrm{m}$, which were higher than the 0.21 - to $0.68-\mu \mathrm{m}$ range measured in the ex vivo eyes without injection [Fig. 3(b)]. The values of the IOP-readout error from the implanted sensors were smaller than $\pm 1 \mathrm{~mm} \mathrm{Hg}$ with an accuracy of $0.58 \pm 0.32 \mathrm{~mm} \mathrm{Hg}$ [Fig. 3(c)], and the absolute values of the IOP error increased with rms-wavefront error, as shown in Fig. 3(d). The factor contributing most to the IOP error was the third-order Zernike term [Fig. 3(e)], which corresponds to comatic and trefoil aberrations. Figure 3(f) shows that the
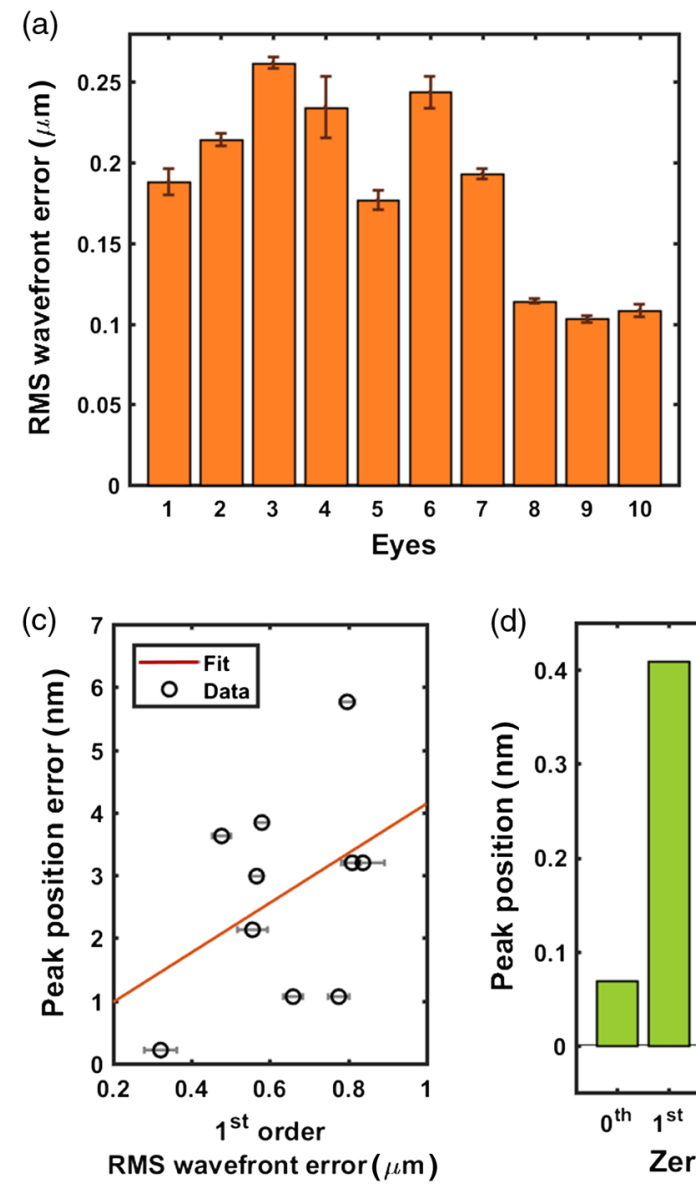

(b)
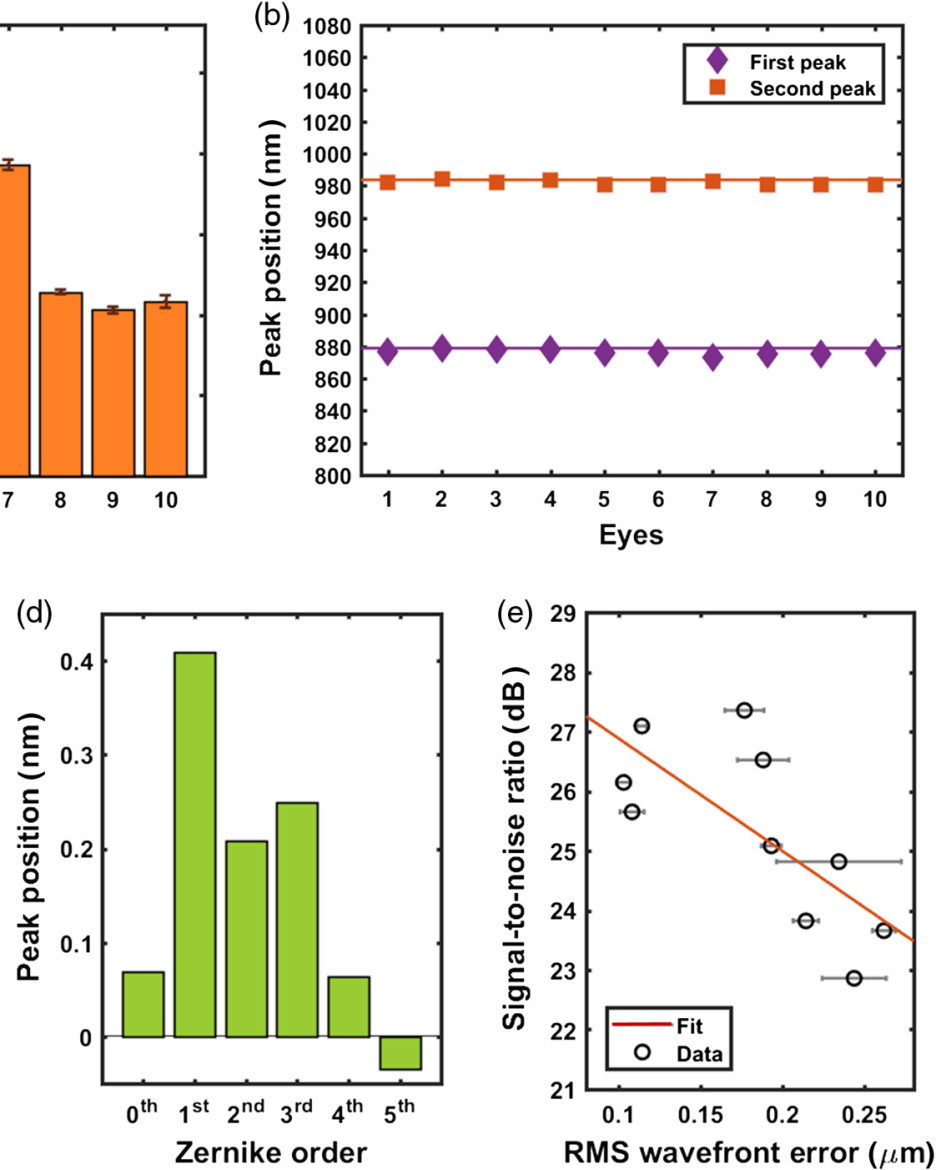

Fig. 2 Measurements of reference chips implanted in 10 ex vivo rabbit eyes. (a) rms-wavefront error of the readouts from the reference chips. (b) Comparison between the peak locations in the optical spectra of a mock chip submerged in water (solid line) and implanted in ex vivo rabbit eyes (squares and diamonds). (c) The absolute errors in the measured peak locations plotted against the first-order Zernike rms-wavefront error. (d) Correlation coefficient of each Zernike order in relation to the error in the measured peak position. (e) SNR of the optical spectra of implanted reference chips plotted against the rmswavefront error. 

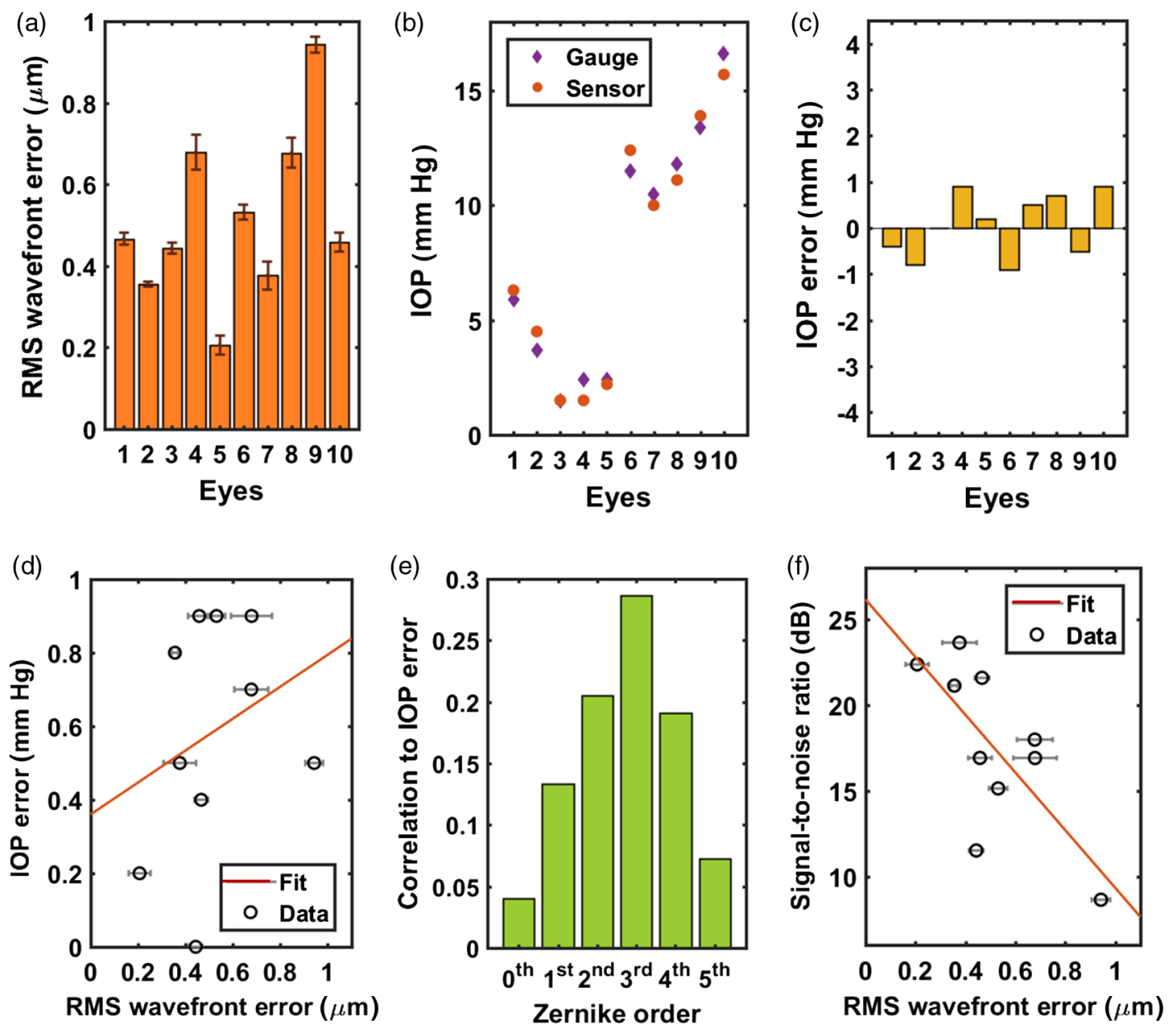

Fig. 3 Aberrations, accuracy, and SNR measured using optical cavity implants in 10 ex vivo rabbit eyes. (a) rms-wavefront errors present in the reflection from implanted IOP sensors. Eyes \#6 to 10 were injected with saline to increase the IOPs. (b) Comparison between the measured IOP values obtained from an implanted IOP sensor and a digital pressure gauge. (c) IOP error in the readouts from an IOP sensor. (d) Absolute IOP error plotted against the rms-wavefront error. (e) Correlation coefficient of each Zernike order in relation to the absolute IOP error. (f) SNR of the optical spectra of an implanted IOP sensor plotted against the rms-wavefront error.

SNR of the optical resonance spectra reflected from the implanted sensors decreased with rms-wavefront error.

\subsection{Accuracy and SNR of Optical Spectra From Optical Cavity Implants at Different Locations}

We also investigated the accuracy of the IOP readouts and the SNR of the optical spectra when the sensors were placed at three different radial positions inside the anterior chamber of an $e x$ vivo rabbit eye. A cross-sectional view of the three sensors placed at different positions is shown in Fig. 4(a). Three IOP sensors were attached to a strip at an interval of $2.5 \mathrm{~mm}$, and the strip with the sensors was inserted through an incision and positioned inside the anterior chamber of an ex vivo rabbit eye [Fig. 4(b)]. The rms-wavefront error measured on each IOP implant is shown in Fig. 4(c). The rms-wavefront error increased with the distance from the implant to the center of the pupil or the optical axis of the eye. The first-order Zernike coefficients contributed the most to the larger rms-wavefront error observed at a greater distance from the center of the pupil [Fig. 4(d)]. The IOP readings from the three implanted sensors and the reference pressure gauge are plotted in Fig. 4(e). The sensor located at the farthest possible distance $(5 \mathrm{~mm})$ from the pupil center showed the largest IOP error of $0.9 \mathrm{~mm} \mathrm{Hg}$ but still remained below $1 \mathrm{~mm} \mathrm{Hg}$, which is the clinically accepted maximum IOP error. In Fig. 4(f), the rms-wavefront error increased with the distance of the sensor from the center; consequently, the SNR of the optical spectra obtained from the IOP sensor decreased but stayed above $15 \mathrm{~dB}$, which was the minimum SNR required for the sensor readout.

\section{Discussion}

\subsection{Influence of Optical Aberrations on Accuracy of Optical IOP Readout}

The magnitudes of the optical aberrations that we characterized in the ex vivo rabbit eyes were very close to the values reported previously in other studies. ${ }^{58}$ Our rms-wavefront errors from all measurements, which lie within $0.37 \pm 0.23 \mu \mathrm{m}$, are slightly larger than previously reported in vivo values, likely because of posthumous distortion of the eye geometry.

In the reference-chip testing, the first-order Zernike coefficients presenting tip and tilt showed the highest correlation 
(a)

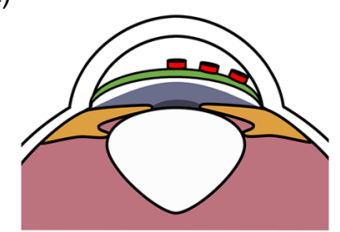

(b)

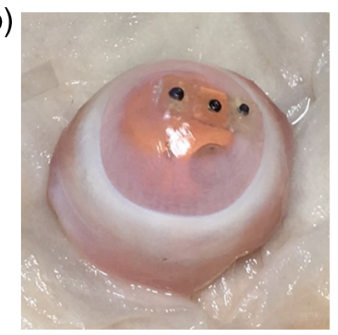

(c)

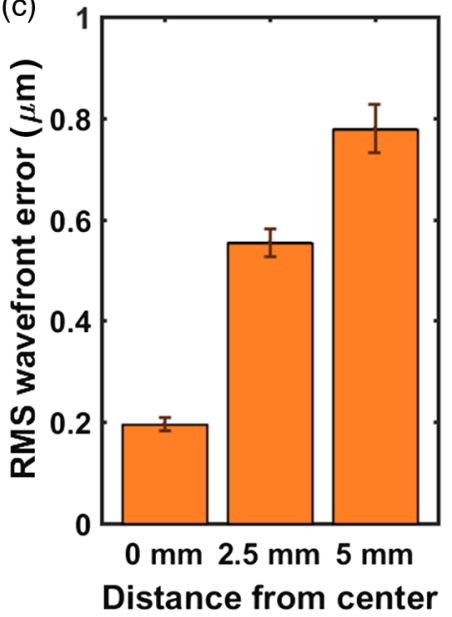

(d)

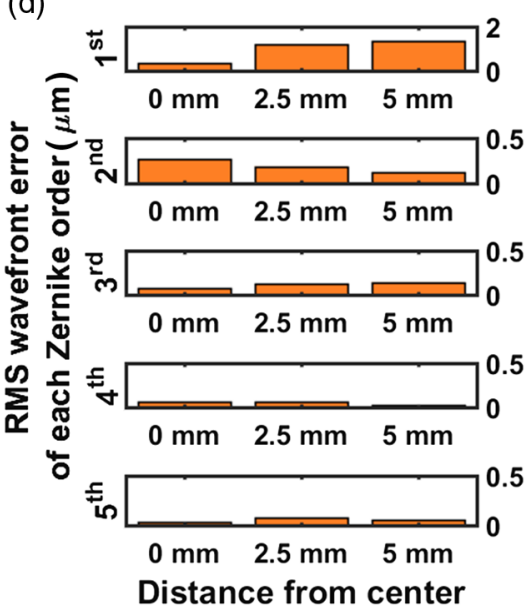

(e)

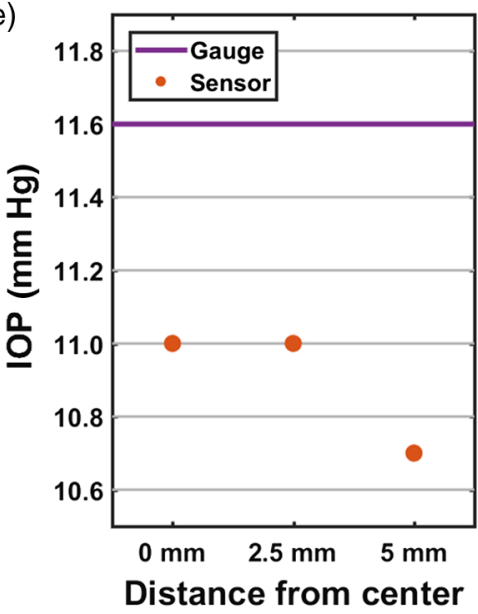

(f)

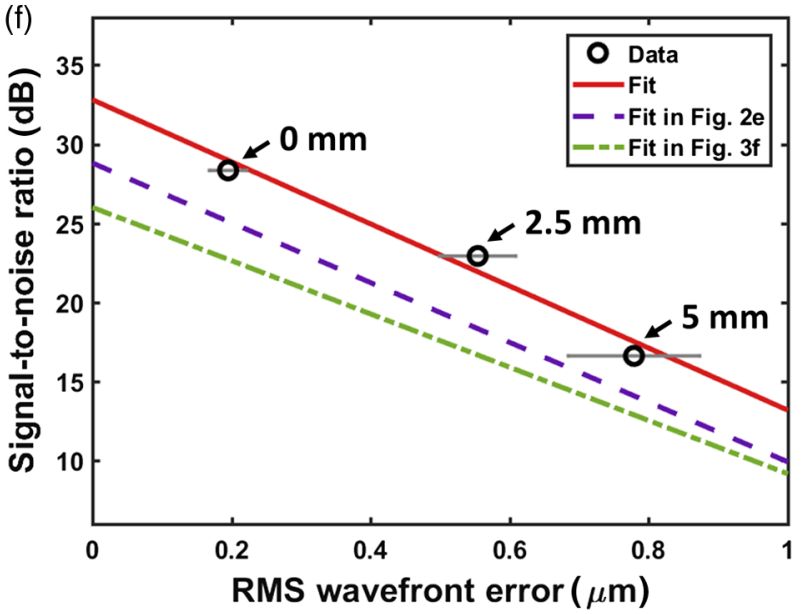

Fig. 4 Measurements using three IOP sensors implanted at three different locations. (a) A cross-sectional illustration of the IOP sensors placed at three different locations. (b) A photograph of the three IOP sensors implanted inside the anterior chamber of an ex vivo rabbit eye. (c) The rms-wavefront error of the readout from each implanted IOP sensor. (d) rms-wavefront error of each Zernike order at different locations. (e) Comparison between the readings from the digital pressure gauge and the implanted IOP sensors. (f) SNR of the optical spectra of the implanted IOP sensors plotted against the rms-wavefront error.

( $r=0.41)$ with erroneous shifts observed in the locations of the resonant peaks [Fig. 2(d)]. This was due to misalignment between the measurement setup and the reference chip. It was challenging to achieve perfect normal incidence between the optical axis of the measurement setup and the surface of the implanted reference chips because the precision of chip placement inside the ex vivo rabbit eyes was limited. The second- (defocus and astigmatism) and third-order (coma) Zernike coefficients were also correlated with the peak-location error, with the third-order coefficient being slightly more dominant. This is again attributed to the fact that defocus and coma are closely related to the precision of the optical alignment. It is also due to the dominance of coma in the native aberration of rabbit eyes. ${ }^{58}$ This outcome is consistent with the principles of thin-film interference or Fabry-Perot cavity resonance; the interference or resonance is heavily dependent on the optical path length, which is determined by the angle of incidence. ${ }^{59}$

A similar trend is observed between the rms-wavefront error of each Zernike order and the readout error of the IOP sensors [Fig. 3(e)], except that the first-order Zernike coefficients, namely tip and tilt, were not as dominant as in reference-chip testing. This indicates that we accomplished better measurement alignment when measuring the IOP sensors. Unlike the implanted reference chips with a $1 \times 1-\mathrm{mm}^{2}$ flat reflective surface, the IOP sensors have a smaller, recessed sensing region made of two different surfaces that tend to be more angle sensitive [Fig. 1(b)]. This angle sensitivity requires better optical alignment during measurements. Therefore, the correlation between the first-order Zernike coefficients and the IOP error became relatively weak. The third-order rms-wavefront error, which represented coma, showed the strongest correlation with the IOP error, and its correlation magnitude of 0.29 was close to the 0.25 observed during the reference-chip measurements [Fig. 2(d)], indicating that the comatic aberrations that we observed in both cases were most likely a part of the native aberrations present in the ex vivo rabbit eyes. ${ }^{58}$ The second most contributing factor for the IOP error was the second-order rmswavefront error, which represents defocus. In addition to the inherent second-order rms-wavefront error to the rabbit cornea, the deformable membrane of the sensor contributed to the 
increase in the second-order rms-wavefront error and its correlation to the IOP error. This trend is consistent with the previous study that showed that the second-order rms-wavefront error originating from a deflected membrane was more sensitive to IOP than the fourth- and higher-order rms-wavefront errors. ${ }^{43}$

When the sensors were implanted at three different radial distances from the pupil center in the anterior chamber [Figs. 4(a) and 4(b)], however, the first-order Zernike coefficients became the dominant factor that increased the total rms-wavefront error [Fig. 4(c)] and the IOP error [Fig. 4(e)]. As the IOP sensor became farther from the center of the pupil, the curvature of the cornea also increased and caused a larger degree of refraction at the interfaces, resulting in significant tip and tilt. Although the third-order Zernike coefficients show the same trend as the first-order ones, the magnitude of the first-order Zernike coefficients is much greater than that of the third-order ones. The dominance of the first-order Zernike coefficients explains the underestimated IOP readouts because of the redshift of the peaks from the sensors positioned farther away from the pupil center. Unlike the first- and third-order terms, the secondand fourth-order rms-wavefront errors decreased at a greater distance from the pupil center. This is partly attributed to the smaller defocus term in the second-order rms-wavefront error as the depth of the sensor location decreased and enabled better focusing. In addition, both the second- and fourth-order coefficients include astigmatism terms, which are negatively correlated with the depth of the sensor location. When a sensor is located closer to the cornea, the focused light illuminates through a smaller area on the cornea, thereby introducing less astigmatism.

\subsection{Influence of Optical Aberrations on SNR of Optical Readout}

The SNR of the optical spectra obtained from the reference-chip measurements was 22.8 to $27.5 \mathrm{~dB}$ [Fig. 2(e)], which exceeds the 7 to $23 \mathrm{~dB}$ of the IOP sensors as shown in Fig. 3(f). This is partly due to the larger measured/reflected area $(1 \mathrm{~mm} \times 1 \mathrm{~mm})$ of the implanted reference chips compared with that of an implanted IOP sensor (0.6 $\mathrm{mm}$ in diameter).

The major factor that decreased the SNR in both cases was the presence of the larger aberrations [Figs. 2(e) and 3(f)]. The larger aberrations came from the implant and/or the biomechanics of ex vivo rabbit eyes. The IOP sensor introduced extra aberration due to its flexible surface geometry. ${ }^{43}$ The native aberration of the sensors $(0.168 \pm 0.014 \mu \mathrm{m})$ accounts for the increase in the average aberration in Fig. 3(b) $(0.514 \mu \mathrm{m})$ when compared with that in Fig. 2(a) $(0.184 \mu \mathrm{m})$. Additionally, the increase in IOP and the variation in the Young's modulus of the corneas could result in the increase in the radius of corneal curvature and the increased aberration. ${ }^{60}$

The reduction in the SNR of the readouts under the greater rms-wavefront errors was observed in both reference-chip testing and IOP-sensor testing as well as in sensor-location testing that involved three sensors implanted at three different radial distances [Fig. 4(g)]. When compared with the SNR of the readouts in reference-chip testing and IOP-sensor testing [Figs. 2(e) and 3(f)], the SNR values obtained from this experiment [Fig. 4(f)] followed a similar fitting line to those used in Figs. 2(e) and 3(f). Furthermore, the dominance of the first Zernike order was observed in the case of sensor-location testing [Fig. 4(d)] but not in the case of IOP-sensor testing [Fig. 3(e)]. These outcomes indicate that the SNR of the readouts was primarily dependent on the total rms-wavefront error and was not particularly dependent on a single Zernike order. With no significant reduction in the SNR near the edge of the anterior chamber, this optical-resonance-based IOP-sensing implant is highly promising for use in human eyes without interrupting the vision.

\section{Conclusion}

We studied the effect of optical aberrations on the accuracy and the SNR of optical IOP monitoring obtained using ocular implants. Both reference chips and optical cavity implants showed high accuracy $(<5.8 \mathrm{~nm}$ for a reference chip and $<1 \mathrm{~mm} \mathrm{Hg}$ with an optical cavity implant) along with good SNR $(25.3 \pm 1.46 \mathrm{~dB}$ for a reference chip and $15.57 \pm 4.85 \mathrm{~dB}$ for an optical cavity implant) over rms-wavefront errors of 0.10 to $0.94 \mu \mathrm{m}$. Additionally, the IOP readout from the sensor that was located radially at $5 \mathrm{~mm}$ from the pupil center where one would expect the largest optical aberrations showed acceptable accuracy and SNR $(<1 \mathrm{~mm} \mathrm{Hg}$ and $>15 \mathrm{~dB})$. These results indicate that the use of optical cavity implants shows great promise for accurate and easy monitoring of IOP.

\section{Disclosures}

The authors declare that there are no conflicts of interest related to this article. All ex vivo experiments were performed using the rabbit eyes enucleated in a FACTA Humane Certified facility (Pel-Freeze).

\section{Acknowledgments}

This work was supported by the National Institutes of Health (No. EY024582) and the Heritage Medical Research Institute.

\section{References}

1. H. A. Quigley and A. T. Broman, "The number of people with glaucoma worldwide in 2010 and 2020," Br. J. Ophthalmol. 90(3), 262-267 (2006).

2. Y. C. Tham et al., "Global prevalence of glaucoma and projections of glaucoma burden through 2040: a systematic review and meta-analysis," Ophthalmology 121(11), 2081-2090 (2014).

3. H. A. Quigley, "Glaucoma," Lancet 377(9774), 1367-1377 (2011).

4. B. Bengtsson et al., "Fluctuation of intraocular pressure and glaucoma progression in the early manifest glaucoma trial," Ophthalmology 114(2), 205-209 (2007).

5. C. G. De Moraes et al., "Risk factors for visual field progression in treated glaucoma," Arch. Ophthalmol. 129(5), 562-568 (2011).

6. A. Heijl et al., "Reduction of intraocular pressure and glaucoma progression: results from the early manifest glaucoma trial," Arch. Ophthalmol. 120(10), 1268-1279 (2002).

7. G. Z. Chen, I. S. Chan, and D. C. Lam, "Capacitive contact lens sensor for continuous noninvasive intraocular pressure monitoring," Sens. Actuators A 203, 112-118 (2013).

8. K. Mansouri and T. Shaarawy, "Continuous intraocular pressure monitoring with a wireless ocular telemetry sensor: initial clinical experience in patients with open angle glaucoma," $\mathrm{Br}$. J. Ophthalmol. 95(5), 627-629 (2011).

9. C. Faschinger and G. Mossböck, "Continuous $24 \mathrm{~h}$ monitoring of changes in intraocular pressure with the wireless contact lens sensor Triggerfish $^{\mathrm{TM}}$. First results in patients," Der Ophthalmologe: Zeitschrift der Deutschen Ophthalmologischen Gesellschaft 107(10), 918-922 (2010).

10. M. Leonardi et al., "A soft contact lens with a MEMS strain gage embedded for intraocular pressure monitoring," in 12th Int. Conf. on TRANSDUCERS, Solid-State Sensors, Actuators and Microsystems, Vol. 2, pp. 1043-1046 (2003). 
11. K. Mansouri et al., "Continuous 24-hour monitoring of intraocular pressure patterns with a contact lens sensor: safety, tolerability, and reproducibility in patients with glaucoma," Arch. Ophthalmol. 130(12), 1534-1539 (2012).

12. M. Greene and B. Gilman, "Intraocular pressure measurement with instrumented contact lenses," Invest. Ophthalmol. Visual Sci. 13(4), 299-302 (1974).

13. M. Leonardi et al., "Wireless contact lens sensor for intraocular pressure monitoring: assessment on enucleated pig eyes," Acta Ophthalmol. 87(4), 433-437 (2009).

14. M. Leonardi et al., "First steps toward noninvasive intraocular pressure monitoring with a sensing contact lens," Invest. Ophthalmol. Visual Sci. 45(9), 3113-3117 (2004).

15. Y. C. Huang et al., "A contact lens sensor system with a microcapacitor for wireless intraocular pressure monitoring," in IEEE Sensors, 2013, pp. 1-4 (2013).

16. N. M. Farandos et al., "Contact lens sensors in ocular diagnostics," $A d v$. Healthcare Mater. 4(6), 792-810 (2015).

17. P. M. Ladage et al., "Pseudomonas aeruginosa corneal binding after 24-hour orthokeratology lens wear," Eye Contact Lens 30(3), 173-178 (2004).

18. M. Imayasu et al., "The relation between contact lens oxygen transmissibility and binding of Pseudomonas aeruginosa to the cornea after overnight wear," Ophthalmology 101(2), 371-388 (1994).

19. D. H. Ren et al., "The relationship between contact lens oxygen permeability and binding of Pseudomonas aeruginosa to human corneal epithelial cells after overnight and extended wear," CLAO J. 25(2), 81 (1999)

20. J. Kim et al., "Wearable smart sensor systems integrated on soft contact lenses for wireless ocular diagnostics," Nat. Commun. 8, 14997 (2017).

21. A. Koutsonas et al., "Implantation of a novel telemetric intraocular pressure sensor in patients with glaucoma (ARGOS study): 1-year results telemetric intraocular pressure sensor," Invest. Ophthalmol. Visual Sci. 56(2), 1063-1069 (2015).

22. A. Todani et al., "Intraocular pressure measurement by radio wave telemetry," Invest. Ophthalmol. Visual Sci. 52(13), 9573-9580 (2011).

23. K. C. Katuri, S. Asrani, and M. K. Ramasubramanian, "Intraocular pressure monitoring sensors," IEEE Sens. J. 8(1), 12-19 (2008).

24. C. C. Collins, "Miniature passive pressure transensor for implanting in the eye," IEEE Trans. Biomed. Eng. BME-14, 74-83 (1967).

25. C. I. Jang et al., "Effects of inner materials on the sensitivity and phase depth of wireless inductive pressure sensors for monitoring intraocular pressure," Appl. Phys. Lett. 108(10), 103701 (2016).

26. Ç. Varel et al., "A wireless intraocular pressure monitoring device with a solder-filled microchannel antenna," J. Micromech. Microeng. 24(4), 045012 (2014).

27. E. Y. Chow, A. L. Chlebowski, and P. P. Irazoqui, "A miniature-implantable RF-wireless active glaucoma intraocular pressure monitor," IEEE Trans. Biomed. Circuits Syst. 4(6), 340-349 (2010).

28. N. Xue, S. P. Chang, and J. B. Lee, "A SU-8-based microfabricated implantable inductively coupled passive RF wireless intraocular pressure sensor," J. Microelectromech. Syst. 21(6), 1338-1346 (2012).

29. K. Stangel et al., "A programmable intraocular CMOS pressure sensor system implant," IEEE J. Solid-State Circuits 36(7), 1094-1100 (2001).

30. L. Rosengren et al., "A system for passive implantable pressure sensors," Sens. Actuators A. 43(1-3), 55-58 (1994).

31. M. H. Ouda et al., "5.2-GHz RF power harvester in 0.18- $\mu \mathrm{m} \mathrm{CMOS}$ for implantable intraocular pressure monitoring," IEEE Trans. Microwave Theory Tech. 61(5), 2177-2184 (2013).

32. A. Donida et al., "A circadian and cardiac intraocular pressure sensor for smart implantable lens," IEEE Trans. Biomed. Circuits Syst. 9(6), 777-789 (2015).

33. P. J. Chen et al., "Microfabricated implantable parylene-based wireless passive intraocular pressure sensors," J. Microelectromech. Syst. 17(6), 1342-1351 (2008).

34. T. Karrock and M. Gerken, "Pressure sensor based on flexible photonic crystal membrane," Biomed. Opt. Express 6(12), 49014911 (2015).

35. R. Melamud et al., "Development of an SU-8 Fabry-Perot blood pressure sensor," in 18th IEEE Int. Conf. on Micro Electro Mechanical Systems (MEMS '05), pp. 810-813 (2005).
36. E. Cibula, D. Donlagic, and C. Stropnik, "Miniature fiber optic pressure sensor for medical applications," in Proc. IEEE Sensors, pp. 711-714 (2002).

37. H. Bae and M. Yu, "Miniature Fabry-Perot pressure sensor created by using UV-molding process with an optical fiber based mold," Opt. Express 20(13), 14573-14583 (2012).

38. G. Hill et al. "SU-8 MEMS Fabry-Perot pressure sensor," Sens. Actuators A 138(1), 52-62 (2007).

39. R. A. Wolthuis et al., "Development of medical pressure and temperature sensors employing optical spectrum modulation," IEEE Trans. Biomed. Eng. 38(10), 974-981 (1991).

40. M. Li, M. Wang, and H. Li, "Optical MEMS pressure sensor based on Fabry-Perot interferometry," Opt. Express 14(4), 1497-1504 (2006).

41. K. Totsu, Y. Haga, and M. Esashi, "Ultra-miniature fiber-optic pressure sensor using white light interferometry," J. Micromech. Microeng. 15(1), 71-75 (2004).

42. A. Phan et al., "Design of an optical pressure measurement system for intraocular pressure monitoring," IEEE Sens. J. 18(1), 61-68 (2018).

43. A. Nazarov et al., "Assessment of intraocular pressure sensing using an implanted reflective flexible membrane," J. Biomed. Opt. 22(4), 047001 (2017).

44. I. E. Araci et al., "An implantable microfluidic device for selfmonitoring of intraocular pressure," Nat. Med. 20(9), 1074-1078 (2014).

45. P. J. Chen et al., "Impanatable micromechanical parylene-based pressure sensors for unpowered intraoular pressure sensing," $J$. Micromech. Microeng. 17(10), 1931-1938 (2007).

46. M. Ghannad-Rezaie et al., "A powerless optical microsensor for monitoring intraocular pressure with keratoprostheses," in Transducers \& Eurosensors XXVII: The 17th Int. Conf. on Solid-State Sensors, Actuators and Microsystems (TRANSDUCERS \& EUROSENSORS XXVII '13), Vol. 17, pp. 2708-2711 (2013).

47. J. O. Lee et al., "A microscale optical implant for continuous in-vivo monitoring of intraocular pressure," Microsys. Nanoeng. 3, 17057 (2017).

48. J. O. Lee et al., "Biocompatible multifunctional black-silicon for implantable intraocular sensor," Adv. Healthcare Mater. 6(4), 1601356 (2017).

49. K. H. Kim et al., "Real-time in vivo intraocular pressure monitoring using an optomechanical implant and an artificial neural network," IEEE Sens. J. 17(22), 7394-7404 (2017).

50. B. C. Platt and R. Shack, "History and principles of Shack-Hartmann wavefront sensing," J. Refract. Surg. 17(5), S573-S577 (2001).

51. P. M. Prieto et al., "Analysis of the performance of the Hartmann-Shack sensor in the human eye," J. Opt. Soc. Am. A 17(8), 1388-1398 (2000).

52. J. Liang et al., "Objective measurement of wave aberrations of the human eye with the use of a Hartmann-Shack wave-front sensor," J. Opt. Soc. Am. A 11(7), 1949-1957 (1994).

53. L. N. Thibos and H. Xin, "Clinical applications of the Shack-Hartmann aberrometer," Optom. Vision Sci. 76(12), 817-825 (1999).

54. A. Guirao et al., "Corneal optical aberrations and retinal image quality in patients in whom monofocal intraocular lenses were implanted," Arch. Ophthalmol. 120(9), 1143-1151 (2002).

55. S. Marcos, B. Sergio, and J. A. Ignacio, "Optical quality and depth-offield of eyes implanted with spherical and aspheric intraocular lenses," J. Refract. Surg. 21(3), 223-235 (2005).

56. F. Taketani et al., "Influence of intraocular lens tilt and decentration on wavefront aberrations," J. Cataract Refractive Surg. 30(10), 2158-2162 (2004).

57. L. N. Thibos et al., "Standards for reporting the optical aberrations of eyes," J. Refractive Surg. 18(5), S652-S660 (2002).

58. L. Chen et al., "Comparison of wavefront aberrations in rabbit and human eyes," Clin. Exp. Optom. 97(6), 534-539 (2014).

59. M. C. Teich and B. Salech, Fundamentals of Photonics, 22nd ed., Wiley Interscience, New York (1991).

60. J. Liu and C. J. Roberts, "Influence of corneal biomechanical properties on intraocular pressure measurement: quantitative analysis," J. Cataract Refractive Surg. 31(1), 146-155 (2005).

Samuel J. Han is currently pursuing his BS degree in bioengineering at the University of Maryland, College Park. He was an Amgen scholar and summer undergraduate research fellow at the California Institute of Technology in 2016 and 2017. His research 
interests include micro-/nanoscale medical devices as well as novel point-of-care diagnostic and therapeutic biomedical technologies that promote human health, especially in resource-limited settings.

Haeri Park is a $\mathrm{MS} / \mathrm{PhD}$ candidate in medical engineering at the California Institute of Technology. She received a BS degree from the Seoul National University in electrical engineering. Her interests include implantable biomedical devices, optics, and photonics. Currently, her research focuses on applying principles of optics and nanophotonics to implantable medical devices.

Jeong Oen Lee received his BS, MS, and PhD degrees in electrical engineering from the Korea Advanced Institute of Technology and completed his postdoctoral training in electrical engineering and medical engineering at the California Institute of Technology. Currently, he is a postdoctoral researcher at the National Institute of Health. His research interests include micro-/nanoscale devices in biomedical sensing, development of implantable sensors and monitoring systems, and neural probing.

Hyuck Choo received his BS and MEng in electrical engineering from Cornell University and his $\mathrm{PhD}$ in electrical engineering and computer sciences from the UC Berkeley, where he also completed his postdoctoral training in conjunction with the UC San Francisco. Currently, he is an assistant professor in electrical engineering and medical engineering at California Institute of Technology, developing innovative medical technologies based on micro-/nanoscale science and engineering to address major health problems. 